\section{ÖRG 2012 - Jahrestagung der ÖRG}

gemeinsam mit:

13. Jahrestagung der AG Kinderradiologie/OERG

Herz-Intensiv-Kurs / Teil 1 der AG Cardiale Bildgebung/OERG

Lehrgang zum ÖNORM zertifizierten MR Sicherheitsbeauftragten

37. Jahrestagung des Verband für Medizinischen Strahlenschutz in Österreich

Verband der RadiologietechnologInnen Österreich

13--15. September 2012 in Graz

\section{Sehr geehrte Kolleginnnen und Kollegen \\ $\nabla$}

Im Namen der ÖRG dürfen wir Sie sehr herzlich zum ÖRGKongress 2012, der vom 13.15. September in Graz stattfindet, einladen. Gemäß dem Tagungsmotto „Inter- und Multidisziplinäre Radiologie, Zukunftsweisende Trends“ möchten wir das Fachgebiet Radiologie in seinem breiten klinischen Stellenwert, aber auch in seinen neuen Facettierungen abbilden. Dies in Form von Fortbildungen, Fit für den Facharzt-Kursen, einem Crashkurs Herz, einem MR-Sicherheitskurs und einer Vortragsserie von RadiotechnologInnen. Neue Konzepte zur Frühdiagnose und ehestmöglichen, personalisierten Therapie im Kontext mit den fortschreitenden Entwicklungen in allen klinischen Fachgebieten erfordern die Diskussion und Anpassung etablierter Abläufe. Auch die zunehmende Verwendung von „Imaging Biomarkern“ und die rasanten Fortschritte in der Medizintechnik verlangen zur Erfüllung der multifaktoriellen Ansprüche der Radiologie neue Formen der fächerübergreifenden Kooperationen zur effizienteren PatientInnenversorgung. Die Bildgebung, die interventionellen Eingriffe und die zunehmend erwartete Unmittelbarkeit der Arzt/Patienten-Beziehungen erfordern hohe medizinische, technische, aber auch ethische Expertise. Wir freuen uns, Sie in Graz, der europäischen Kulturhauptstadt 2003, aber auch einem renommierten Wissenschaftsstandort mit 4 Universitäten begrüßen zu dürfen. Veranstaltungsort ist das MUMUTH - Haus für Musik und Musiktheater, das in unmittelbarer räumlicher wie sachbezogener Zuordnung zur Kunst-

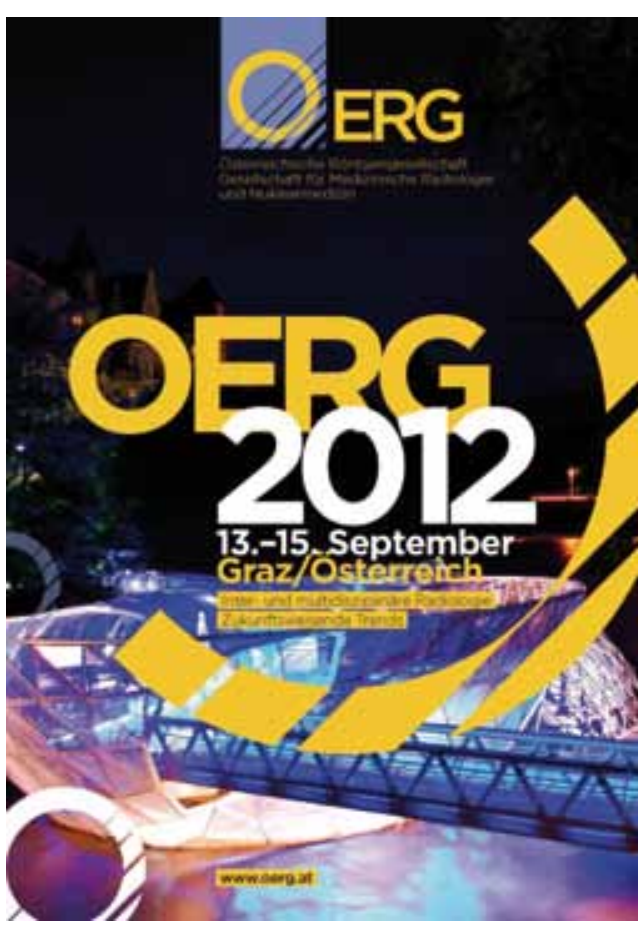
universität Graz steht. MUMUTH als Schnittstelle zwischen Universität und Gesellschaft, als innovative Plattform für einen Diskurs der Moderne, aber auch als unverwechselbares Veranstaltungsgebäude trägt dem universitären Auftrag entsprechend Entwicklung und Erschließung der Künste sowie wissenschaftlicher Forschung vollumfänglich Rechnung.

In der Hoffnung Ihr geschätztes Interesse geweckt zu haben und auf ein Wiedersehen in Graz!

Univ.-Prof. ${ }^{\text {in }}$ Dr. ${ }^{\text {in }}$ Reingard M. Aigner, Kongresspräsidentin

ao. Univ.-Prof. Dr. Helmut Schöllnast, Kongresssekretär

ao. Univ.-Prof. Dr. Erich Sorantin, Kongresssekretär

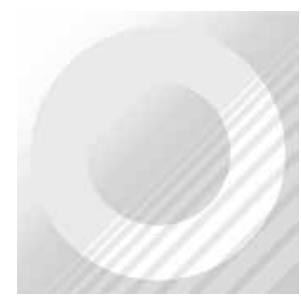

\section{Programmschwerpunkte}

$\nabla$

- Onkologische Radiologie und Intervention im Abdomen

$\checkmark$ Intervention

- Neurointervention

- Morphologische und funktionelle Diagnostik in der Endokrinologie

- Hybrid Imaging

- Kinderradiologie

- Muskuloskeletale Erkrankungen

- Traumatische Erkrankungen in der Osteoradiologie

\section{Wichtige Daten}

$\nabla$

2. Juli: Ende der Einreichfrist für wissenschaftliche Beiträge

- 25. Juli: Verständigung der Autoren über die Annahme der Beiträge

1. August: Anmeldeschluss für ermäßigte Teilnahmegebühren

$\checkmark 5$. September: Ende der Voranmeldefrist zum Kongress

- 13. September: Öffnung des Anmeldeschalters im MUMUTH

\section{Einladung zur Einreichung wissen- schaftlicher Beiträge|Abstracts $\nabla$}

Das Tagungspräsidium lädt Sie herzlich ein, wissenschaftliche Beiträge zur Präsentation einzureichen. Informationen zur Einreichung erhalten Sie auf der Website www.oerg.at.

\section{Beiträge für freie Vorträge}

Bitte beachten Sie, dass Ihr Beitrag in der vorliegenden Form noch nicht publiziert worden sein darf und einschließlich Titel, Autoren und Adresse maximal 250 Wörter umfassen darf. Längere Texte werden automatisch gekürzt. Folgende Gliederung müssen Sie beachten:

> Problemstellung

> Patienten und Methoden

$>$ Ergebnisse

- Schlussfolgerungen

Beiträge für „Jugend präsentiert..." Interessante, lehrreiche Fälle können in interdisziplinären Sitzungen präsentiert werden. Diese Abstracts dürfen ein- 
schließlich Titel, Autoren und Adresse maximal 100 Wörter umfassen und sind wie folgt gegliedert:

> Patienten und Methoden

- Schlussfolgerungen

Nur Beiträge, die den Richtlinien entsprechen, werden an die Begutachter weitergeleitet. Alle angenommenen Beiträge (mit Ausnahme von „Jugend präsentiert ...") werden im Organ der Gesellschaft, der Zeitschrift „RöFo”, Thieme Verlag, in zitierbarer Form publiziert. Dazu ist eine Anmeldung zum Kongress notwendig.
Tagungspräsidium

$\nabla$

- Univ.-Prof. ${ }^{\text {in }}$ Dr. ${ }^{\text {in }}$ med. Reingard Aigner, Kongresspräsidentin Universitätsklinik für Radiologie Auenbruggerplatz 9, A-8036 Graz

- Univ.-Prof. H. Schöllnast, Graz/AT

- Univ.-Prof. E. Sorantin, Graz/AT

\section{Kongressbüro}

$\nabla$

Sekretariat OERG 2012, c/o ESR Büro

Neutorgasse $9 / 2 a$

A-1010 Wien

Tel.: +43/1/5320507

Fax: +43/1/5320507448

E-Mail: kongress@oerg.at

\section{Hotelbuchung}

Graz Tourismus und Stadtmarketing $\mathrm{GmbH}$

Messeplatz 1/Messeturm

A-8010 Graz

Tel.: +43/316/ 807542

E-Mail: af@graztourismus.at

\section{Tagungsort}

MUMUTH - Haus für Musik und

Musiktheater

Lichtenfelsgasse 14

8010 Graz

\begin{tabular}{|c|c|c|c|c|}
\hline \multicolumn{5}{|c|}{ Vorprogramm Donnerstag, 13. September 2012} \\
\hline & György-Ligeti-Saal & Proberaum & Studiobühne & Klinikum \\
\hline 08:00-08:15 & & \multirow{4}{*}{$\begin{array}{l}\text { Einführung } \\
\text { in die } \\
\text { Facharztprüfung }\end{array}$} & & \\
\hline 08:15-08:30 & & & & \\
\hline 08:30-08:45 & & & & MR-Sicherheitskurs I \\
\hline 08:45-09:00 & & & & \\
\hline 09:00-09:15 & \multirow[t]{4}{*}{ Kongresseröffnung } & & & \\
\hline 09:15-09:30 & & & & MR-Sicherheitskurs II \\
\hline 09:30-09:45 & & & & \\
\hline 09:45-10:00 & & & & \\
\hline 10:00-10:15 & \multirow[t]{2}{*}{ Kaffeepause } & & & \\
\hline 10:15-10:30 & & & & \\
\hline 10:30-10:45 & \multirow[t]{6}{*}{ Interventionen } & \multirow{6}{*}{$\begin{array}{l}\text { Fit für den } \\
\text { Facharzt I } \\
\text { Kraniozerebrale } \\
\text { Erkrankungen I }\end{array}$} & & MR-Sicherheitskurs III \\
\hline 10:45-11:00 & & & & \\
\hline 11:00-11:15 & & & & \\
\hline $11: 15-11: 30$ & & & & MR-Sicherheitskurs IV \\
\hline $11: 30-11: 45$ & & & & \\
\hline 11:45-12:00 & & & & \\
\hline 12:00-12:15 & & & & \\
\hline $12: 15-12: 30$ & \multirow[t]{4}{*}{ Lunchsymposium } & & & \\
\hline $12: 30-12: 45$ & & & & \\
\hline 12:45-13:00 & & & & \\
\hline 13:00-13:15 & & & & \\
\hline 13:15-13:30 & & & & \\
\hline $13: 30-13: 45$ & \multirow[t]{6}{*}{ Neurointerventionen } & \multirow{6}{*}{$\begin{array}{l}\text { Fit für den } \\
\text { Facharzt II } \\
\text { Kraniozerebrale } \\
\text { Erkrankungen II/ Spinale } \\
\text { Erkrankungen I }\end{array}$} & \multirow{6}{*}{$\begin{array}{l}\text { wissenschaftliche } \\
\text { Sitzung }\end{array}$} & MR-Sicherheitskurs V \\
\hline $13: 45-14: 00$ & & & & \\
\hline $14: 00-14: 15$ & & & & \\
\hline 14:15-14:30 & & & & MR-Sicherheitskurs I \\
\hline 14:30-14:45 & & & & \\
\hline $14: 45-15: 00$ & & & & \\
\hline 15:00-15:15 & \multirow[t]{2}{*}{ Kaffeepause } & & & \\
\hline 15:15-15:30 & & & & \\
\hline $15: 30-15: 45$ & \multirow{6}{*}{$\begin{array}{l}\text { Onkologische } \\
\text { Radiologie } \\
\text { und } \\
\text { Interventionen } \\
\text { im } \\
\text { Abdomen }\end{array}$} & \multirow{6}{*}{$\begin{array}{l}\text { Fit für den } \\
\text { Facharzt II } \\
\text { Spinale } \\
\text { Erkrankungen II }\end{array}$} & \multirow{6}{*}{$\begin{array}{l}\text { wissenschaftliche } \\
\text { Sitzung }\end{array}$} & MR-Sicherheitskurs VII \\
\hline $15: 45-16: 00$ & & & & \\
\hline $16: 00-16: 15$ & & & & \\
\hline 16:15-16:30 & & & & MR-Sicherheitskurs VIII \\
\hline $16: 30-16: 45$ & & & & \\
\hline $16: 45-17: 00$ & & & & \\
\hline 17:00-17:15 & & & & \\
\hline $17: 15-17: 30$ & \multirow{4}{*}{$\begin{array}{l}\text { ÖRG } \\
\text { Jahreshauptversammlung }\end{array}$} & & & \\
\hline $17: 30-17: 45$ & & & & \\
\hline 17:45-18:00 & & & & \\
\hline 18:00-18:15 & & & & \\
\hline 18:15-18:30 & & & & \\
\hline 18:30 & \multicolumn{3}{|l|}{ Begrüßungsabend } & \\
\hline
\end{tabular}




\begin{tabular}{|c|c|c|c|c|}
\hline \multicolumn{5}{|c|}{ Vorprogramm Freitag, 14. September 2012} \\
\hline & György-Ligeti-Saal & Proberaum & Studiobühne & Klinikum \\
\hline \multicolumn{5}{|l|}{ 08:00-08:15 } \\
\hline \multicolumn{5}{|l|}{$08: 15-08: 30$} \\
\hline 08:30-08:45 & \multirow[t]{6}{*}{ Mammografie } & \multirow{6}{*}{$\begin{array}{l}\text { Fit für den } \\
\text { Facharzt IV } \\
\text { Kinderradiologie }\end{array}$} & \multirow{6}{*}{$\begin{array}{l}\text { Crashkurs } \\
\text { Herz }\end{array}$} & \multirow[t]{3}{*}{ MR-Sicherheitskurs IX } \\
\hline 08:45-09:00 & & & & \\
\hline 09:00-09:15 & & & & \\
\hline 09:15-09:30 & & & & MR-Sicherheitskurs X \\
\hline 09:30-09:45 & & & & \\
\hline 09:45-10:00 & & & & \\
\hline $10: 00-10: 15$ & \multirow[t]{2}{*}{ Kaffeepause } & & & \\
\hline $10: 15-10: 30$ & & & & \\
\hline $10: 30-10: 45$ & \multirow{6}{*}{$\begin{array}{l}\text { Hybrid } \\
\text { Imaging }\end{array}$} & \multirow{6}{*}{$\begin{array}{l}\text { Fit für den } \\
\text { Facharzt V } \\
\text { Muskuloskelettale } \\
\text { Erkrankungen }\end{array}$} & \multirow{6}{*}{$\begin{array}{l}\text { Crashkurs } \\
\text { Herz }\end{array}$} & \multirow[t]{3}{*}{ MR-Sicherheitskurs XI } \\
\hline $10: 45-11: 00$ & & & & \\
\hline $11: 00-11: 15$ & & & & \\
\hline $11: 15-11: 30$ & & & & \multirow[t]{3}{*}{ MR-Sicherheitskurs XII } \\
\hline $11: 30-11: 45$ & & & & \\
\hline $11: 45-12: 00$ & & & & \\
\hline $12: 00-12: 15$ & & & & \\
\hline $12: 15-12: 30$ & \multirow[t]{4}{*}{ Lunchsymposium } & & & \\
\hline $12: 30-12: 45$ & & & & \\
\hline $12: 45-13: 00$ & & & & \\
\hline $13: 00-13: 15$ & & & & \\
\hline \multicolumn{5}{|l|}{$13: 15-13: 30$} \\
\hline $13: 30-13: 45$ & \multirow[t]{6}{*}{ Kinderradiologie } & \multirow{6}{*}{$\begin{array}{l}\text { Fit für den } \\
\text { Facharzt VI } \\
\text { Traumatische } \\
\text { Erkrankungen } \\
\text { in der } \\
\text { Osteoradiologie }\end{array}$} & \multirow{6}{*}{$\begin{array}{l}\text { Crashkurs } \\
\text { Herz }\end{array}$} & \multirow[t]{3}{*}{ MR-Sicherheitskurs XIII } \\
\hline $13: 45-14: 00$ & & & & \\
\hline $14: 00-14: 15$ & & & & \\
\hline $14: 15-14: 30$ & & & & MR-Sicherheitskurs XIV \\
\hline $14: 30-14: 45$ & & & & \\
\hline $14: 45-15: 00$ & & & & \\
\hline $15: 00-15: 15$ & \multirow[t]{2}{*}{ Kaffeepause } & & & \\
\hline $15: 15-15: 30$ & & & & \\
\hline $15: 30-15: 45$ & \multirow{6}{*}{$\begin{array}{l}\text { PET-CT } \\
\text { und } \\
\text { MR-PET } \\
\text { des } \\
\text { Thorax }\end{array}$} & \multirow{6}{*}{$\begin{array}{l}\text { Fit für den } \\
\text { Facharzt VII } \\
\text { Entzündliche } \\
\text { Erkrankungen } \\
\text { des } \\
\text { Bewegungsapparats }\end{array}$} & \multirow{6}{*}{$\begin{array}{l}\text { Crashkurs } \\
\text { Herz }\end{array}$} & \multirow[t]{3}{*}{ MR-Sicherheitskurs XV } \\
\hline $15: 45-16: 00$ & & & & \\
\hline $16: 00-16: 15$ & & & & \\
\hline $16: 15-16: 30$ & & & & \multirow[t]{3}{*}{ MR-Sicherheitskurs XVI } \\
\hline $16: 30-16: 45$ & & & & \\
\hline $16: 45-17: 00$ & & & & \\
\hline $17: 00-17: 15$ & & & & \\
\hline $17: 15-17: 30$ & & Jugend & & \\
\hline $17: 30-17: 45$ & & präsentiert ... & & \\
\hline $17: 45-18: 00$ & & & & \\
\hline $18: 00-18: 15$ & & & & \\
\hline
\end{tabular}

\begin{tabular}{|c|c|c|c|c|}
\hline \multicolumn{5}{|c|}{ Vorprogramm Samstag, 15. September 2012} \\
\hline & György-Ligeti-Saal & Proberaum & Studiobühne & Klinikum \\
\hline \multicolumn{5}{|l|}{ 08:00-08:15 } \\
\hline \multicolumn{5}{|l|}{$08: 15-08: 30$} \\
\hline $08: 30-08: 45$ & \multirow{6}{*}{$\begin{array}{l}\text { Tagung } \\
\text { Verband der } \\
\text { Radiologie- } \\
\text { technologlnnen } \\
\text { Österreich }\end{array}$} & \multirow{6}{*}{$\begin{array}{l}\text { Jahrestagung } \\
\text { Verband für } \\
\text { medizinischen } \\
\text { Strahlenschutz } \\
\text { Österreichs }\end{array}$} & \multirow{6}{*}{$\begin{array}{l}\text { Fit für den } \\
\text { Facharzt } \\
\text { VIII } \\
\text { Osteoradiologie }\end{array}$} & \\
\hline 08:45-09:00 & & & & \\
\hline 09:00-09:15 & & & & \\
\hline 09:15-09:30 & & & & \\
\hline 09:30-09:45 & & & & \\
\hline \multicolumn{2}{|l|}{$09: 45-10: 00$} & & & \\
\hline 10:00-10:15 & \multirow[t]{2}{*}{ Kaffeepause } & & & \\
\hline $10: 15-10: 30$ & & & & \\
\hline $10: 30-10: 45$ & \multirow{6}{*}{$\begin{array}{l}\text { Der } \\
\text { radiologische } \\
\text { Befund }\end{array}$} & \multirow{6}{*}{$\begin{array}{l}\text { Jahrestagung } \\
\text { Verband für } \\
\text { medizinischen } \\
\text { Strahlenschutz } \\
\text { Österreichs }\end{array}$} & \multirow{6}{*}{$\begin{array}{l}\text { Morphologische } \\
\text { und } \\
\text { funktionelle } \\
\text { Diagnostik } \\
\text { in der } \\
\text { Endokrionologie }\end{array}$} & \\
\hline $10: 45-11: 00$ & & & & \\
\hline $11: 00-11: 15$ & & & & \\
\hline $11: 15-11: 30$ & & & & \\
\hline $11: 30-11: 45$ & & & & \\
\hline $11: 45-12: 00$ & & & & \\
\hline
\end{tabular}

